\title{
Long-term retinotopic priming in object identification
}

\author{
SEAN P. MCAULIFFE and BARBARA J. KNOWLTON \\ University of Califormia, Los Angeles, Califormia
}

\begin{abstract}
An important result in perception research is that priming in an object naming task is invariant with translation and left-right reflection. A more sensitive object recognition paradigm was used in three experiments in order to investigate the extent to which priming of object identification is affected by changes in left-right orientation and position. In a prime phase, participants viewed consecutively presented object images. In a subsequent probe phase, participants identified familiar objects in rapid visual streams of nonobject distractors. In Experiment 1, images previously viewed in the same left-right orientation were primed more than images previously viewed in the opposite orientation (i.e., a left-right reflection). This reflection-sensitive priming was replicated in Experiment 2 using a brief (300-msec) prime exposure. In Experiment 3, when the retinal locations of prime and probe images matched, reflection-sensitive priming was also obtained, but when the retinal locations of prime and probe images differed, no reflection-sensitive priming was observed. These results suggest that a single prime exposure can produce long-term priming that is sensitive to left-right reflection, but that this priming is specific to a retinal location.
\end{abstract}

Objects previously viewed can be identified faster and more accurately than objects not previously viewed (Bartram, 1974; Biederman \& Cooper, 1991a, 1991b, 1992). Presumably, viewing the object on the first (prime) occasion activates a representation that is easier to reactivate on the subsequent (probe) occasion. Priming is important in that it may reveal the properties of the underlying representations that mediate object recognition. For example, it has been shown that priming in object identification tasks is invariant with translation and left-right orientation of the object image (Biederman \& Cooper, 1991a). These results suggest that the shape representations that mediate object recognition are also invariant with translation and left-right orientation.

In many studies of the long-term memory representations of object shape, it has been assumed that only the highest level (e.g., object) shape representations are primed over the long term (i.e., more than a few minutes), although there have been exceptions (Srinivas, 1993). Brain areas involved in early visual processing are seen as perceptual in that they construct representations that can be matched with those in memory. However, this conceptualization of the early visual areas as a completely erasable scratchpad may be incorrect; there is evidence to suggest that learning can occur that is specific to retinal locations (Rivest, Boutet, \& Intrilligator, 1997; Shiu \&

S.P.M. was supported by an individual NIMH National Research Service Award (MH 12183). The authors wish to thank Thomas Sanocki and Pepper Williams for their comments on the manuscript, and Brandi Bailey and Kimloan Tran for research assistance. Correspondence concerning this article should be addressed to B. J. Knowlton, Department of Psychology, Franz Hall, University of California, Los Angeles, CA 90095 (e-mail: knowlton@psych.ucla.edu).
Pashler, 1992). Since early visual areas map to specific retinal locations, it is likely that retinally specific learning reflects learning in early visual areas.

In the present study, we investigated the possibility that object recognition might be affected by long-term priming occurring in early visual brain areas. If priming did occur in early visual areas, it would likely exhibit two important properties. First, we would expect such priming to be retinotopic because early visual representations are mapped to specific retinal locations (Hubel \& Wiesel, 1959). Second, early visual priming would be sensitive to a left-right reflection of the image, because the receptive fields of early visual areas (e.g., neurons in V1) are sensitive to feature orientation (and therefore sensitive to leftright reflection).

Given that previous studies had not demonstrated any significant effects of position and left-right reflection, why did we expect to find such effects? Although the results of Biederman and Cooper (1991a) did not support the existence of statistically significant retinotopic priming for object recognition, they did find a nonsignificant suggestion of retinotopic priming. Biederman and Cooper (1991a) observed a fairly consistent numerical advantage of about $5 \mathrm{msec}$ for probe objects appearing in a previously seen location. This advantage for objects appearing in the same location might have been due to retinotopic priming of low-level visual representations, but the variance of the naming task might have prevented the advantage from being statistically significant.

In the present study, we used a new visual identification paradigm designed to provide a more sensitive estimate of visual priming. In the priming phase, subjects viewed object images in a visual stream. In the first block of probe trials, the subjects named a familiar object appearing in a 


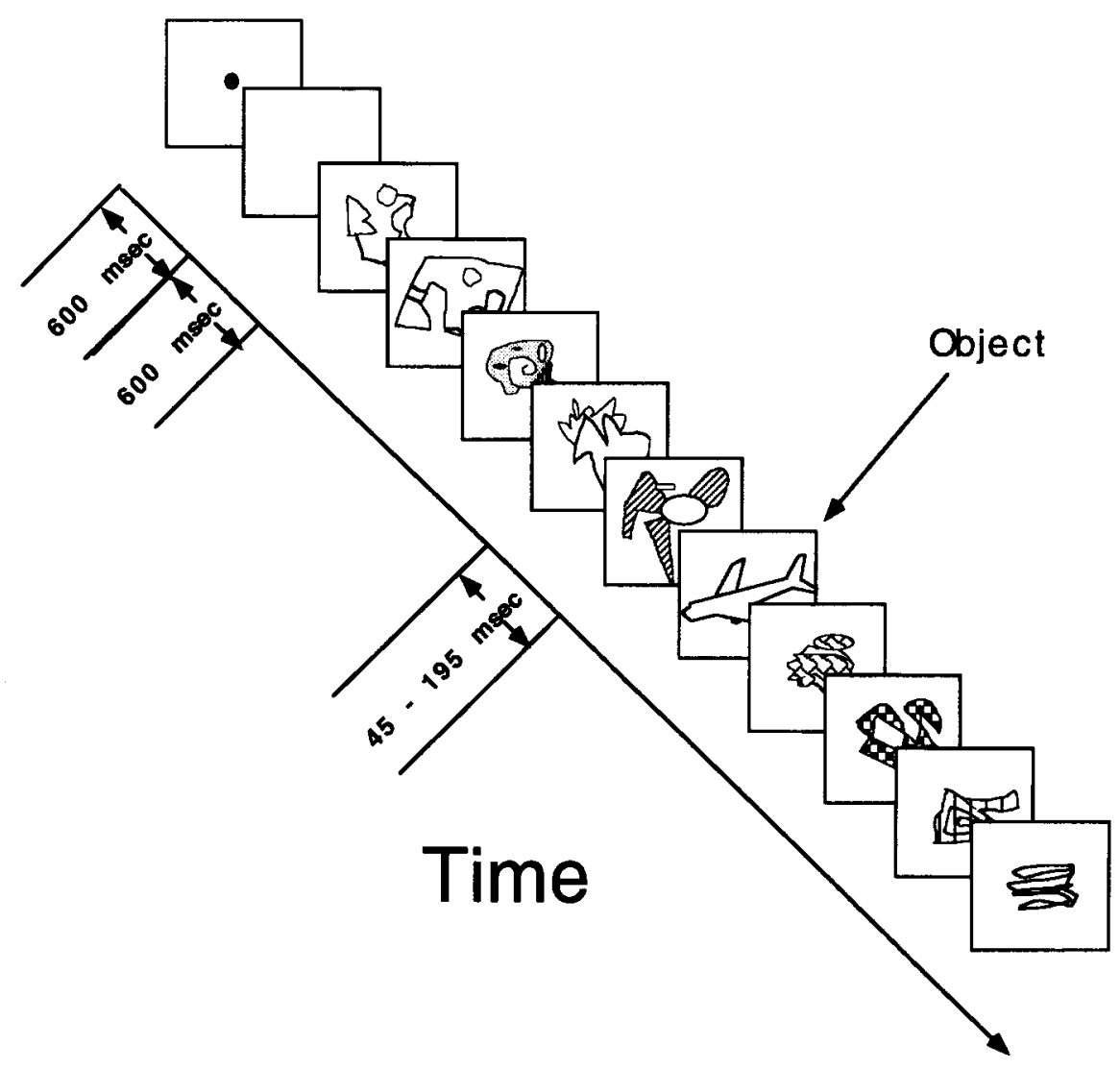

Figure 1. Presentation sequence for a probe trial.

rapid serial visual streams of nonobject distractors (Figure 1) with an extremely brief (i.e., 45-msec) stimulus onset asynchrony (SOA). At this SOA, object images were very difficult to name. All objects not named in this first block were presented in a second block of trials, in which stream SOAs were increased slightly (i.e., $30 \mathrm{msec}$ ). This staircase procedure continued until an SOA was reached (i.e., $225 \mathrm{msec}$ ) at which virtually all the objects could be named. The SOA at which an object was first named served as the dependent variable, which provided an estimate of the processing time necessary to name each object. This procedure results in much lower variance than that produced by naming response times, because much of the variance in a naming task is related to the production of the naming response. (The standard errors in Experiments 1 and 2 reported here average to be less than $3 \mathrm{msec}$ with 12 subjects; the standard errors in Experiment 1 of Biederman and Cooper [1992], who used a naming response time, averaged to be about 6 or $7 \mathrm{msec}$ with 64 subjects.)

In Experiment 1, subjects named images in the priming phase (SOA: $1,200 \mathrm{msec}$ ) depicting familiar objects that varied in the prime-probe relationship (identical vs. leftright reflected version). Presumably, if long-term priming occurred that was reflection sensitive, the probes previ- ously seen in the same version should be named with shorter SOAs than should probes previously seen in a left-right reflected version. Experiment 2 replicated Experiment 1 , but with a shorter prime exposure duration $(300 \mathrm{msec})$ to minimize the effects of episodic representations that have been found sensitive to left-right reflection (Cooper, Schacter, Ballesteros, \& Moore, 1992). Experiment 2 also included an unprimed baseline so that the size of reflectional sensitive priming could be calculated in comparison with the size of visual priming that is reflectionally invariant (Biederman \& Cooper, 1991 a). In Experiment 3, we investigated the effects of a change in retinal position on long-term reflection-sensitive priming. The subjects named objects presented to either the left or the right of fixation in the priming phase and then identified objects as they appeared in one of two visual streams in the left and right locations. Presumably, if reflection sensitive long-term priming arises from early visual areas, it should occur only when probe and prime images are presented in the same retinal location.

\section{EXPERIMENT 1}

In Experiment 1, we investigated the effects of leftright reflection on long-term priming of visual represen- 
tations. We hypothesized that identical probes previously seen should be identified at shorter durations than probes previously seen in a left-right reflected version.

\section{Method}

Subjects. Twelve undergraduates at the University of California at Los Angeles participated to fulfill a course requirement. All participants had normal or corrected-to-normal vision.

Design. We used a two-level design, with condition (identical primed, reflected-version primed) as a within-subjects variable.

Materials. The stimuli were black line drawings on a white background, presented on a Macintosh color monitor. All of the drawings depicted objects in a prototypical view that was asymmetrical about the vertical axis. For example, a horse would be viewed from the side, with the head facing to the left or to the right. The distractor images were generally bounded surfaces but also contained texture information in some cases. Similar to the test images, the distractor images were also asymmetrical about the vertical axis. The entire experimental session was controlled by a program written in the Macprobe (Aristometrics) experiment programming language. The subjects viewed the display binocularly from a distance of approximately $70 \mathrm{~cm}$, and object images subtended approximately $2.6^{\circ}$ of visual angle.

Procedure. All subjects were tested individually. To reduce the variability in the names that the subjects gave for various objects, all subjects first read aloud all the object names from a piece of paper. The experimenter then read instructions to the subject. The subjects were instructed to name the images that would appear sequentially on the screen. The prime displays began with the appearance of a filled circle in the center of the screen; this circle remained on for $1,000 \mathrm{msec}$. A white screen was then displayed for $500 \mathrm{msec}$, followed by a visual stream of line drawings with each image appearing for $1,200 \mathrm{msec}$. The subjects' naming responses were recorded on tape.

Each probe trial presented a rapid stream of images (one object image among 17 nonobject distractors), and the subject's task was to identify the object. The experimenter entered the first three letters of the subject's response into the computer, and the computer determined whether the subject's response was correct or incorrect by comparing these letters with the first three letters of the correct response.

Figure 1 illustrates the sequence of images on a single probe trial. Each trial began with the appearance of a fixation oval, displayed for $1,000 \mathrm{msec}$, followed by a blank screen for $500 \mathrm{msec}$. Seventeen distractor images were randomly chosen from a set of 24 and were displayed sequentially. The probe image appeared in a serial position, randomly chosen from Positions 7-13. The images subtended approximately $2.6^{\circ}$ of visual angle and were presented at fixation. In the first block of the probe phase, all images were displayed for $45 \mathrm{msec}$. Any object that the subject could not name in the first block was presented again in the second block. Display times in the second block were $75 \mathrm{msec}$. This procedure was repeated, adding $30 \mathrm{msec}$ to the display time on each successive block, until all objects had been identified or a display time of $250 \mathrm{msec}$ was reached. Objects that could not be identified in or before the 250 -msec block were treated as errors. In all, there were 48 probe images, plus six practice trials (given at the beginning of the probe phase) depicting objects not appearing in either the prime or the probe trials. Across all subjects, each object was equally likely to appear in each of the 2 conditions. With 48 objects and 2 conditions, there were 24 objects in each cell per subject.

\section{Results}

In the identical condition, the mean display time needed for recognition was $67.5 \mathrm{msec}(S E M=3.6)$ and in the left-right reflected condition, the mean display time needed for recognition was $71.6 \mathrm{msec}(S E M=3.3)$. A repeated measures $t$ test showed the difference between these two conditions to be significant $[t(11)=2.51, p<$ $.05)$. There were no errors, in that each subject identified all objects with display times of $250 \mathrm{msec}$ or less.

\section{Discussion}

As predicted, objects previously named in the identical version were identified at shorter durations than were objects previously named in a left-right reflected version. These results depart from the widely held view that longterm priming of objects is unaffected by left-right reflection. As suggested in the introduction, there were some hints in previous studies of such an effect; but the use of high-variance tasks (e.g., naming) may have prevented the detection of this identical-version advantage.

\section{EXPERIMENT 2}

The results of Experiment 1 support the idea that longterm reflection-sensitive priming can occur and that this priming appears to be retinotopic. Given that these results stand in contrast to those of a number of previous studies (Biederman \& Cooper, 1991a; Cooper, Biederman, \& Hummel, 1992), in Experiment 2 we sought to replicate this finding, using a shorter display time in the priming phase. The $1,200-\mathrm{msec}$ duration might have allowed the creation of episodic memories for the visual stimulus. By episodic memory, we refer to a representation that contains the temporal context of the item's presentation. These representations would be accessed when items are judged as having been seen before. It has been shown that such representations are reflection sensitive, because subjects are less likely to judge an item as old if it is presented again as a reflected version (Cooper, Schacter, et al., 1992). Therefore, Experiment 2 replicated Experiment 1, but with a 300-msec exposure duration for each prime image. Potter (1969) demonstrated that episodic encoding is significantly reduced with shorter exposure durations (e.g., $300 \mathrm{msec}$ ). Therefore, with the short exposure duration of Experiment 2, the influence of episodic representations should be minimized. The probe phase in Experiment 2 was identical to the probe phase in Experiment 1.

Experiment 2 also included an unprimed baseline condition to measure the magnitude of priming that might exist in both the identical and the left-right conditions. Use of the unprimed baseline condition to calculate absolute priming was important, because it would reveal the size of the reflectional sensitive priming (i.e., the priming observed in Experiment 1) in comparison with the size of the visual priming which is reflection invariant. Probe objects appearing in the unprimed baseline condition did not appear in the priming phase. As in Experiment 1 , probe objects in the identical condition appeared 
exactly as they had appeared in the prime phase, and probe objects in the left-right reflected condition appeared in a left-right reflected version (i.e., a mirror reflection).

\section{Method}

Subjects. Twelve undergraduates from the University of California at Los Angeles participated to fulfill a course requirement. All subjects had normal or corrected-to-normal vision.

Design. In this experiment, we used a three-level design, with condition (identical primed, reflected-version primed, and unprimed baseline) as a within-subjects variable.

Materials. The stimuli were the same as those in Experiment 1.

Procedure. The procedure was identical to that of Experiment 1, with the following exceptions in the priming phase: The object images were displayed for $300 \mathrm{msec}$ (as opposed to 1,200 msec in Experiment 1), and the subjects were instructed to attend to the objects in preparation for a later memory test but were not asked to name them. With 48 objects and 3 conditions, there were 16 objects in each cell per subject.

\section{Results}

Priming in the identical and the left-right reflected conditions was operationalized by subtracting the mean recognition display times in these conditions from the mean recognition display time in the baseline condition. One subject was excluded from data analysis because that subject could not identify all the objects in the allotted time. All of the other subjects identified all of the objects with display times of $250 \mathrm{msec}$ or less. In the identical condition, the mean display time needed for recognition was $76.4 \mathrm{msec}(S E M=2.1)$ and in the left-right reflected condition, the mean display time needed for recognition was $80.9 \mathrm{msec}(S E M=1.8)$, and in the baseline condition, the mean display time needed for recognition was $88.0 \mathrm{msec}(S E M=2.4)$. The data reveal $11.5 \mathrm{msec}$ of priming in the identical condition, which a repeated measures $t$ test showed to be significantly greater than the $7.1 \mathrm{msec}$ of priming in the left-right reflected condition $[t(11)=2.49, p<.05]$. Both the identical condition and the left-right reflected conditions produced priming that was significantly greater than zero $[t(11)=5.76, p<$ $.001]$ for the identical condition and $[t(11)=4.06, p<$ $.01]$ for the left-right reflected condition.

\section{Discussion}

As in Experiment 1, objects previously seen in the identical version were identified at shorter durations than objects previously seen in a left-right reflected version. These results offer further support for the existence of long-term reflection-sensitive priming of visual representations. In addition, this experiment demonstrated reflection-invariant priming, although some of the reflection-invariant priming may have been nonvisual (e.g., greater semantic priming may have occurred with image naming than in the unprimed condition, where only the word was read).

Whereas previous studies (Biederman \& Cooper, 1991a) have reported significant reflection-invariant priming and no reflection-sensitive priming, in the present study we report reflection-sensitive priming that is not only detectable, but also relatively large in comparison with the reflection-invariant priming observed. In specific, the identical-version prime resulted in a display time of $76.4 \mathrm{msec}$, whereas the left-right reflected prime resulted in $80.9 \mathrm{msec}$. This $4.5 \mathrm{msec}$ of priming is about $60 \%$ as large as the $7.1 \mathrm{msec}$ of reflection-invariant priming. The sensitivity of the present paradigm may be responsible for the detection of the reflection-sensitive priming. In addition, the short exposure duration in Experiment 2 $(300 \mathrm{msec})$ might have not been sufficient for complete activation (and therefore priming) of reflection-invariant representations. Indeed, there is evidence that viewpoint sensitivity diminishes over the course of several hundred milliseconds (Kourtzi \& Shiffrar, 1996). These two factors together produce reflection-sensitive priming that is relatively large in comparison with reflection-insensitive priming.

\section{EXPERIMENT 3}

In Experiments 1 and 2, objects previously seen in the identical version were identified at shorter durations than were objects previously seen in a left-right reflected version. In Experiment 3, we examined the effects of a change in retinal position on this identical-version advantage. If the identical version priming advantage observed in Experiments 1 and 2 was retinotopic, only probe objects presented in the same (prime) retinal location should show an identical-version advantage. Conversely, if this identical version priming advantage was not retinotopic, the identical-version priming advantage should persist despite a change in the retinal position of the object image.

Experiment 3 differed from the first two experiments in that objects were not presented in a visual stream. In each trial, subjects attended to a fixation point in the center of the screen and then the object appeared on either the left or the right side of fixation. In this way, we ensured that the object would appear in the appropriate retinal location. We feared that a rapid sequence of objects presented laterally might produce saccades that would compromise the retinal location manipulation.

In the probe phase, two visual streams were presented instead of one. In the probe task, subjects identified an object image appearing in one of the two rapid visual streams of nonobject distractors. Again, a staircase procedure was used, in which images were repeated until a display time was reached at which virtually all the objects could be identified.

\section{Method}

Subjects. Twenty undergraduates at the University of California at Los Angeles participated to fulfill a course requirement. All subjects had normal or corrected-to-normal vision. Nineteen subjects were right-handed and one was left-handed.

Design. In this experiment, we used a 5 (identical primed/ identical position vs. identical primed/opposite position vs. reflected 


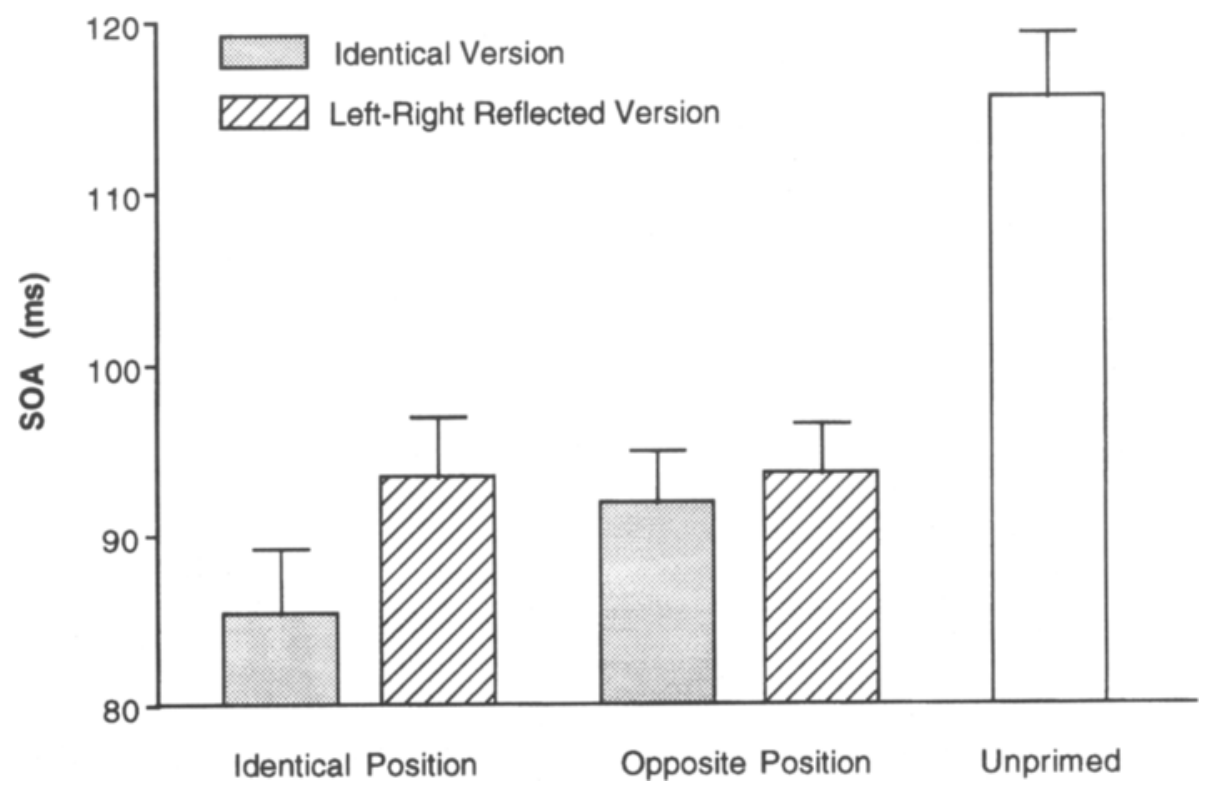

Figure 2. Experiment 3: Minimum stimulus onset asynchrony (SOA) necessary for identification as a function of prime version (identical vs. left-right reflected) and prime position (identical vs. opposite).

primed/identical position vs. reflected primed/opposite position vs. unprimed baseline) $\times 10$ (object group) design, with both factors as within-subjects variables.

Materials. The stimuli were the same as those used in Experiment 1, except that 50 objects were used.

Procedure. As in Experiments 1 and 2, all subjects first read aloud all the object names from a piece of paper. The experimenter then read instructions to the subjects. In the prime phase, the subjects attended to a fixation point for $180 \mathrm{msec}$. After $500 \mathrm{msec}$ of a blank screen, either an object name (unprimed condition) or an object image (primed conditions) appeared on the screen. The object names were presented centrally in Helvetica 60 font and were read by the subjects. For the primed conditions (identical version/same location, reflected version/same location, identical version/different location, reflected version/different location), the subjects were instructed to name the object image. The object images appeared $4^{\circ}$ to the left of fixation or $4^{\circ}$ to the right of fixation for $180 \mathrm{msec}$. The objects appeared for $180 \mathrm{msec}$ to avoid the possibility of a saccade. In the probe phase, the object images appeared in two visual streams that were centered $4^{\circ}$ from a fixation point that remained on the screen throughout the probe trial. The subjects were instructed that an object image representing a nameable object (e.g., a tree) would appear in one of two visual streams of distractors (e.g., nonnameable objects). The subjects were instructed to divide their attention equally between both streams while maintaining their gaze at the fixation point. In the first block of the probe phase, an image appeared in one visual stream (randomly chosen to be either left or right) for $30 \mathrm{msec}$, and then the next image would appear in the other visual stream for $30 \mathrm{msec}$. This alternating sequence would continue for all images in the stream. This procedure produced an SOA of 60 msec, because for each visual stream, 30-msec images were interleaved with blank screens of equal durations. Any objects not identified in the first block were presented again in the second block for $45 \mathrm{msec}$ (producing an SOA of $90 \mathrm{msec}$ ). This procedure was repeated, adding $15 \mathrm{msec}$ to the display time (and $30 \mathrm{msec}$ to the SOA) until all objects were identified or until the display time was $135 \mathrm{msec}$ (with an SOA of $270 \mathrm{msec}$ ). Objects that could not be identified in a stream of items with an SOA of 270 msec were treated as errors.

Each subject identified 50 probe images. The objects were counterbalanced across subjects so that each object image appeared equally in each of the 5 conditions (identical version/same location, reflected version/same location, identical version/different location, reflected version/different location, and unprimed) and in each of the 2 probe positions (left vs. right visual field). Therefore, each subject viewed 10 ( 5 conditions $\times 2$ probe positions) object groups with 5 objects in each group. In addition, half the subjects viewed a group of images that were left-right reflections of the images viewed by the other half of subjects. Therefore, a complete counterbalanced set included 20 subjects ( 10 object groups $\times 2$ left-right reflections). With 50 objects and five conditions, there were $10 \mathrm{ob}-$ jects in each cell per subject.

\section{Results}

Figure 2 shows the mean SOAs necessary to recognize the probe objects in all conditions. A 5 (unprimed, identical position/identical version, identical position/reflected version, opposite position/identical version, opposite poisition/reflected version, and unprimed) $\times 10$ (object group) ANOVA revealed a significant effect of condition $[F(4,40)=30.14, p<.0001]$. The effect of object group was not significant $[F(9,10)=2.72, p>.05]$. A NewmanKeuls post hoc test revealed that all of the primed conditions had significantly shorter identification times than did the unprimed condition, in which the name of the object was read in the prime phase $(p<.001)$. In addition, the identical version/identical position condition ( $85.3 \mathrm{msec}, S E M=3.9$ ) required significantly shorter SOAs than did each of the other three primed conditions $(p<.05$ for reflected version/identical position [93.3 msec, $S E M=3.5] ; p<.05$ for identical version/op- 
posite position [ $91.7 \mathrm{msec}, S E M=3.0$ ]; $p<.05$ for reflected version/ opposite position [ $93.5 \mathrm{msec}, S E M=2.9]$ ). Mean error rates were low (identical version/identical position $=0.0 \%$; reflected version $/$ identical position $=1.0 \%$, $S E M=.6 \%$; identical version/opposite position $=2.0 \%$, $S E M=.9 \%$; reflected version/opposite position $=1.0 \%$, $S E M=.6 \%$; unprimed $=1.5 \%, S E M=.8 \%$ ) and there were no significant differences among the error rates for any of the conditions $[F(4,76)=1.23, p>.05]$.

\section{Discussion}

When prime and probe retinal positions were the same, objects previously named in the identical version were identified with shorter display times than were objects previously named in a left-right reflected version. However, when prime and probe retinal positions differed, objects previously named in the identical version were identified as fast as objects previously named in a left-right reflected version. These results suggest that the identicalversion advantage observed in Experiments 1 and 2 reflects priming in low-level (i.e., retinotopic) representations, because a change in retinal position eliminates the advantage for the same version. Conversely, it is unlikely that this identical priming advantage reflects the activation of high-level representations.

The reflectional and location-sensitive priming observed in Experiment 3 was numerically larger (i.e., about 6-8 $\mathrm{msec}$ ) than the priming observed in Experiments 1 and 2 (i.e., about 4-5 msec). This difference may have been due to the increased difficulty of the probe task in Experiment 3. In the reflected-version condition, the mean display times that subjects needed to identify object images in Experiment 3 was about $92 \mathrm{msec}$, whereas object images were identified in about $72 \mathrm{msec}$ in Experiment 1 and $81 \mathrm{msec}$ in Experiment 2. With the more difficult and time-consuming task of Experiment 3, there was greater opportunity for primed representations to facilitate processing.

The priming that did not depend on reflection or retinal location was also much larger in Experiment 3 (about $21-23 \mathrm{msec}$ ) than it was in Experiment 2 (about $7 \mathrm{msec}$ ). This may have been the result of two factors. First, as was the case with reflectional and location-sensitive priming, the increased difficulty of the probe task in Experiment 3 (115-msec SOA for the unprimed condition vs. 88 -msec SOA for the unprimed condition for Experiment 2) may have allowed more room for priming to affect processing. Second, the degree of prime processing was likely to be greater for Experiment 3, because primes were named with an interstimulus interval of several seconds. In contrast, primes were presented in Experiment 2 for only $300 \mathrm{msec}$, and naming was not required. Thus, the combination of a stronger prime trace and a more difficult probe task may have both contributed to the larger reflection-invariant priming effect observed in Experiment 3.

\section{GENERAL DISCUSSION}

In three experiments, we investigated the effects of left-right reflection and retinal position on long-term object identification priming. In Experiments 1 and 2, when the retinal location of prime and probe images matched, objects previously presented in the identical version were identified at a shorter duration than were objects previously presented in a left-right reflected version. In Experiment 3, when prime and probe retinal positions were the same, objects previously presented in the identical version were identified at a shorter duration than objects previously presented in a left-right reflected version. However, when prime and probe retinal positions differed, objects previously presented in the identical version were identified as fast as objects previously presented in a left-right reflected version.

While the results reported here suggest that long-term left-right specific priming can occur, the precise retinal specificity of this priming remains an open question. From the present study, we can conclude that the left-right specific priming does not cross the midline, which suggests that this priming resides at a level of $\mathrm{V} 4$ or below because the receptive fields in V4 do not generally cross the midline (McKeefry \& Zeki, 1997; Schiller \& Lee, 1991). A more precise manipulation of retinal position will determine whether the priming observed in the present study resides primarily in V4 or in earlier visual areas such as V1 or V2. A computational analysis might suggest that the priming is unlikely to be completely retinotopic, because such priming might quickly saturate the visual system. It is possible that the priming observed in this study is similar to the priming observed by Bar and Biederman (1999), which transfers within a visual quadrant but not between quadrants. Indeed, a next logical step in the present line of study will be to investigate the effects of within-quadrant priming (i.e., V4) versus identical position priming (i.e., V1).

How can the present findings be reconciled with previous studies showing no identical-version priming advantage? It is likely that the increased sensitivity of the present paradigm (relative to naming response time paradigms) may have been a major factor in the detection of the observed effects. The variance of the dependent variable in this study (i.e., image display times) is several times smaller than the variance of the dependent variables used in naming studies (Biederman \& Cooper, 1991a). In addition, Cooper, Schacter, et al. (1992) observed a numerical but nonsignificant reflection-specific priming advantage using a possible/impossible judgment task in which novel objects were presented in the prime phase. Therefore, the results of the present study do have some support in previous research.

The present results have important implications for the study of visual representations. The existence of long- 
term retinotopic priming suggests that low-level visual representations (existing perhaps in the primary visual cortex) can be primed over the long term. These results argue against the idea that the early visual areas function as a completely erasable scratchpad, in that a single exposure produced priming that lasted many minutes. In fact, this long-term priming can occur with even a brief exposure $(1,200 \mathrm{msec}$ in Experiment 1, and only $300 \mathrm{msec}$ in Experiment 2). Instead, the result suggests that longterm priming and thus implicit learning probably occur at almost all levels of the visual hierarchy-providing support for the idea that perception and memory are highly interactive.

The results of the present study also have important implications for understanding the reflection sensitivity (and ultimately the structure) of the visual representations that support object recognition. It has long been assumed in object recognition priming studies that longterm priming reflected priming only of higher level representations. The results reported here provide evidence against that assumption. Therefore, inferences about the shape representations drawn from priming studies must incorporate the fact that both low-level and high-level priming may be present if the retinal locations of the prime and the probe image do not differ.

Ultimately, a complete understanding of object recognition must involve an elucidation of the representational properties at each level of the visual hierarchy. The results of this study suggest that researchers must consider the relationship between prime and probe locations after deciding which level of the visual hierarchy is to be investigated. Specifically, when one is studying the properties of higher level shape representations, prime and probe images should be presented in different retinal locations, thereby minimizing the influence of low-level priming, because these low-level representations are more location specific. When one is studying the properties of lower level shape representations, prime and probe images should be presented in the same retinal locations, thereby maximizing the influence of low-level priming, because these low-level representations are more location specific.

In summary, the results reported here suggest that long-term retinotopic priming can occur from a single brief prime exposure. In addition to highlighting the existence of learning early in the visual system, the results have important implications for the study of object shape representations at various levels in the visual system.

\section{REFERENCES}

BAR, M., \& BiEDERMAN, I. (1999). Localizing the cortical region mediating visual awareness of object identity. Proceedings of the National Academy of Sciences, 96, 1790-1793.

BARTRAM, D. J. (1974). The role of visual and semantic codes in object naming. Cognitive Psychology, 6, 325-356.

Biederman, I., \& Cooper, E. (1991a). Evidence for complete translational and reflectional invariance in visual object priming. Perception, 20. 585-593.

Biederman, I., \& Cooper, E. (1991b). Priming contour-deleted images: Evidence for intermediate representations in visual object recognition. Cognitive Psychology, 23, 393-419.

Biederman, I., \& CoOPER, E. (1992). Size invariance in visual object priming. Journal of Experimental Psychology: Human Perception \& Performance, 18, 121-133.

CoOper, E., Biederman, I., \& Hummel, J. (1992). Metric invariance in object recognition: A review and further evidence. Canadian Journal of Psychology, 46, 191-214.

Cooper, L., Schacter, D., Ballesteros, S., \& Moore, C. (1992). Priming and recognition of transformed three-dimensional objects: Effects of size and reflection. Journal of Experimental Psychology: Learning, Memory, \& Cognition, 18, 43-57.

HuBEL, D. H., \& WIESEL. T. N. (1959). Receptive fields of single neurons in the cat's striate cortex. Journal of Physiology, 148, 574-591.

Kourtzi, Z., \& Shiffrar, M. (1996). From view dependency to view invariance: A spatiotemporal continuum. Abstracts of the 3rd Annual Meeting of the Cognitive Neuroscience Society, 3, 123.

MCKeEFRY, D., \& ZEKI, S. (1997). The position and topography of the human colour centre as revealed by functional magnetic resonance imaging. Brain, 120, 2229-2242.

Potter, M. (1969). Recognition memory for a rapid sequence of pictures. Journal of Experimental Psychology, 81, 10-15.

Rivest, J., Boutet, I., \& INTRILligator, J. (1997). Perceptual learning of orientation discrimination by more than one attribute. Vision Research, 37, 273-281.

SCHILLER, P., \& LEE, K. (1991). The role of the primate extrastriate area $\mathrm{V} 4$ in vision. Science, 251, 1251-1253.

SHIU, L.-P., \& PASHLER, H. (1992). Improvement in line orientation discrimination is retinally local but dependent on cognitive set. Perception \& Psychophysics, 52, 582-588.

SRINIVAS, K. (1993). Perceptual specificity in nonverbal priming. Journal of Experimental Psychology: Learning, Memory, \& Cognition, $19,582-602$.

(Manuscript received June 16, 1998; revision accepted for publication July 21,1999 .) 